Of those patients commenced on LDTA; 10 (83\%) entered clinical remission. 2 (17\%) failed due to lack of clinical response and commenced MTX. 10 remain in a sustained clinical remission at a mean length of follow up of 16.2 months (range: 4-23).

Of the 10 patients commenced on MTX; 7 (70\%) failed due to lack of clinical response and $1(10 \%)$ due to side-effects (Pneumonitis). Of those patients that failed; 4 (40\%) underwent colectomy, 2 (20\%) received arsenic suppositories and entered a sustained clinical remission. $2(20 \%)$ were reclassified to Crohn's disease, were treated with biologic therapy and entered a sustained clinical remission. Mean length of follow up in this group was 17.6 months (range: 2-30).

Conclusion Thiopurines remain the mainstay of treatment for patients with UC. A significant number of patients fail this conventional treatment and represent a clinical challenge. Novel treatments such as LDTA can be effective in a significant proportion of this group. Data for the efficacy of MTX remains less effective and topical arsenic is useful and can be helpful.

Disclosure of Interest T. Hollingworth: None Declared, H. JOHNSON Conflict with: SPONSORSHIP FROM FALK, ABBOTT \& WARNER CHILCOTT TO ATTEND MEETINGS, R. BASUROY: None Declared, S. MCLAUGHLIN Conflict with: SPONSORSHIP FROM FALK TO ATTEND MEETINGS, S. WEAVER Consultant for: MSD ADVISORY BOARD, Conflict with: SPONSORSHIP FROM FALK, ABBOT, MSD \& FERRING TO ATTEND MEETINGS

\section{PTH-116 MICROSCOPIC COLITIS IN TAYSIDE - FURTHER OBSERVATIONS ON CLINICAL FEATURES AND OUTCOME}

doi:10.1136/gutjnl-2013-304907.603

1,"T Heron, ${ }^{1} \mathrm{C}$ Mowat, ${ }^{1} \mathrm{M}$ Groome, ${ }^{2} \mathrm{~S}$ Walsh, ${ }^{1} \mathrm{~S}$ Siddhi, ${ }^{1} \mathrm{~A}$ Meritsi. 'Gastroenterology; ${ }^{2}$ Pathology, NHS Tayside, Dundee, UK

Introduction The aetiology of microscopic colitis remains unknown. We have previously reported our experience of microscopic colitis diagnosed in our region between 1999 - 2004(1). Although data continue to emerge, the natural history of microscopic colitis remains unclear. Furthermore, there are reports of macroscopic changes in the mucosa at endoscopy.

Methods Cases from 2004-2011 were identified from pathology records. Case notes were retrospectively reviewed and data extracted including subtype of microscopic colitis and clinical details where possible.
Results 82 case notes were obtained and reviewed. 8 cases did not have a clear diagnosis and were excluded. Of the remaining 74 cases, 56 were collagenous colitis, 18 lymphocytic colitis. 17 patients had macroscopic abnormalities (excluding diverticulosis) at endoscopy; 16 of these in the collagenous colitis group, representing $28 \%$ of this subgroup. The mean age was 46.1 (range 33-87), female: male ratio of 4.3:1. 18 reported an autoimmune condition including 2 coeliac disease and 7 hypothyroidism. 30(40\%) were on a proton pump inhibitor at the time of diagnosis and $15(24 \%)$ were on non-steroidal anti-inflammatories. Follow up data was available for 66 patients. Of these $47(71 \%)$ reported complete resolution of symptoms and $15(22 \%)$ partial resolution. $5(7 \%)$ did not respond in the follow up period. Therapeutic strategies included either alone or a combination of stopping/switching PPI, loperamide, mesalazine and steroids. $24 / 47(65 \%)$ of complete responders required simple intervention (PPI withdrawal, switch in brand of PPI, loperamide or even spontaneous resolution) whereas 10/47(21\%) required steroids. $7 / 15(47 \%)$ partial responders received steroids.

Conclusion Since microscopic colitis was last studied in our region, the female predominance has increased, the mean age has dropped by almost $20 y r s$, and the ratio of collagenous : lymphocytic colitis has increased from $2: 1$ to $3: 1$. This could represent a change in the number of younger people investigated or missing data in our cohort. A significant number of patients with a diagnosis of collagenous colitis had endoscopic abnormalities in comparison to the lymphocytic colitis group, which does raise the question of the nomenclature. The majority of patients have complete resolution of symptoms with simple intervention.

Disclosure of Interest None Declared.

\section{REFERENCE}

1. Microscopic Colitis in Tayside: Clinical features, associations and behaviour. Mowat C, Heron T, Walsh S. Gastroenterology 2005: 128 (4); A331

\section{PTH-117 PREDICTING THE NEED FOR DOSE ESCALATION IN PATIENTS WITH CROHN'S DISEASE TREATED WITH ADALIMUMAB}

doi:10.1136/gutjnl-2013-304907.604

1." $\mathrm{V}$ C Kariyawasam, ' $\mathrm{M}$ G Ward, ${ }^{2} \mathrm{~V}$ Collings, ' ${ }^{1} \mathrm{~K}$ Patel, ${ }^{1} \mathrm{R}$ Goel, ${ }^{1} \mathrm{P}$ A Blaker, ${ }^{1} \mathrm{~S}$ Anderson, 'J D Sanderson, 'P M Irving. 'Gastroenterology; ${ }^{2}$ Pharmacy, Guy's and St Thomas' NHS Foundation Trust, London, UK

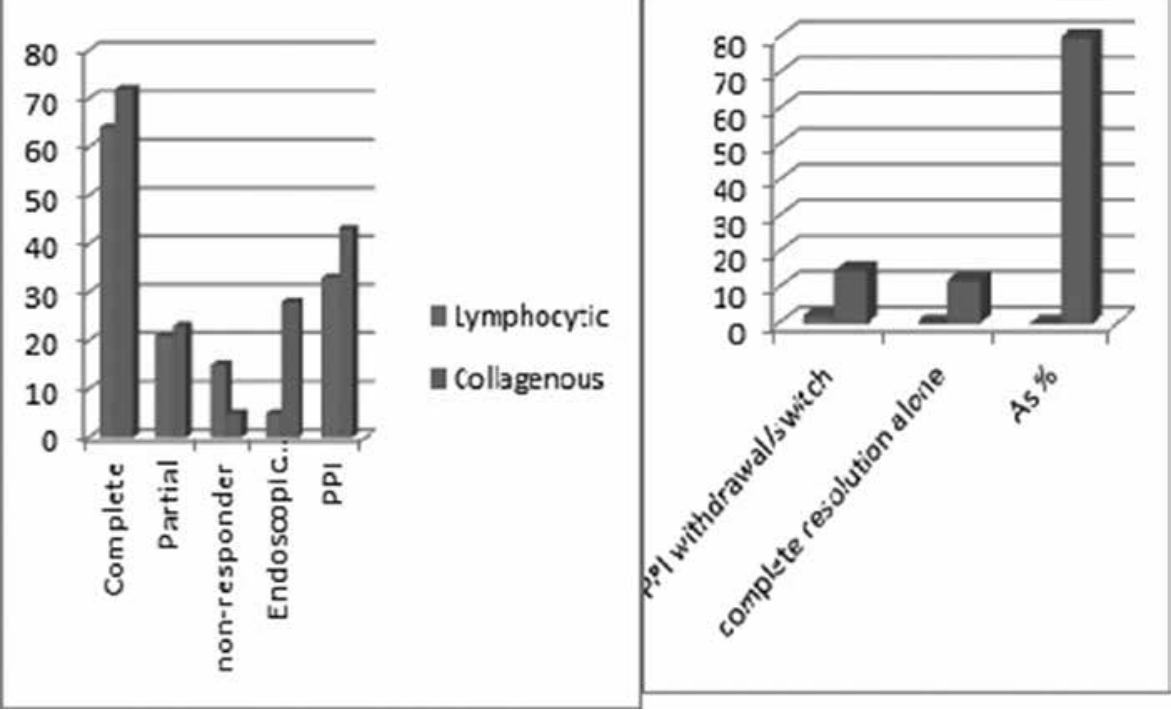

Abstract PTH-116 Figure 1 
Introduction Adalimumab ( $\mathrm{ADA}$ ) is effective for the induction and maintenance of remission in Crohn's disease (CD) patients. Although the approved maintenance regimen is $40 \mathrm{mg}$ subcutaneously every 14 days, some patients require dose-escalation (DE).

Methods Aim of the study was to describe a large, well-characterised cohort of $\mathrm{ADA}$ treated $\mathrm{CD}$ patients in a tertiary referral centre and to identify factors predicting the need for DE. A prospectively maintained database of $\mathrm{CD}$ patients treated with $\mathrm{ADA}$ at the Guy's and St Thomas' IBD Center between 2007-2012 was interrogated. Clinical and phenotypic details and exposure to therapy were analysed. Survival and regression analyses were performed.

Results 112 CD (50\% Male) patients commenced ADA. Three patients had coexisting Oro-Facial Granulomatosis. Mean age at diagnosis was 22 (SD; 9) years. Disease location was ileo-colonic $(68.8 \%)$ in the majority. Upper gastrointestinal (UGI) involvement was found in $17.9 \%$, peri-anal disease in $29.5 \%$ and extra-intestinal manifestations in $14.3 \%$.

Median duration of disease prior to ADA initiation was 11 years (IOR; 5-18). Previous infliximab (IFX), ADA and exposure to both were found in 59, 3 and 7 patients respectively. Of the 66 patients exposed to IFX 29 (43.9\%) had primary or secondary loss of response. A total of $82(71.3 \%)$ were on concomitant immunomodulators ((CIM) - azathioprine, mercaptopurine, thioguanine or methotrexate) at the time of initiation of $\mathrm{ADA}$.

103 (89.3) patients responded to ADA induction. 4 patients were primary non-responders, 5 withdrew due to adverse effects. All 4 primary non-responders and $3 / 5$ who withdrew were previously exposed to IFX.

$\mathrm{DE}$ was required in 40 (38.8\%) of the responders during the followup period at a median 26 months (95\% confidence interval (CI); 19.632.4). Cumulative probability of requiring $\mathrm{DE}$ at 24 months was $52 \%$ (CI: 42-62). CIM at initiation of ADA (Odds ratio (OR): 0.21, CI: 0.09-0.47, p<0.0001), previous IFX exposure (OR: 4.27, CI: 1.73-10.55, $\mathrm{p}=002)$ and UGI involvement (OR: 3.43, CI: 1.02-11.42, p = 0.046) were independently associated with need for $\mathrm{DE}$ in multivariate analysis. CIM at commencement of $\mathrm{ADA}$ was associated with increased time to DE (Hazard ratio: 0.34, CI: 0.18-0.64, p=0.001). 24/38 (63.25\%) patients responded to $\mathrm{DE}, 2$ patients had incomplete follow-up data.

Conclusion $38.8 \%$ of $\mathrm{CD}$ patients commencing ADA in this well defined cohort required $\mathrm{DE}$ due to loss of response, of which $63 \%$ were recaptured. CIM at initiation predicted a more durable response to standard dosing. UGI involvement and previous exposure to IFX were associated with increased risk of requiring DE.

Disclosure of Interest None Declared.

\section{Nursing}

\section{PTH-118 QUALITY OF BOWEL PREPARATION FOR COLONOSCOPY: CAN WE LEARN ANYTHING FROM PATIENTS' PERSPECTIVES?}

doi:10.1136/gutjnl-2013-304907.605

\author{
1,"J Alexander, 'S Moore, 'P Neild. 'St George's Hospital, London, UK
}

Introduction Good quality bowel preparation (prep) with oral laxative agents is vital for accurate and safe colonoscopy. Previous work has studied associations between specific patient characteristics and inadequate bowel prep ${ }^{1}$. We analysed patients' perspectives of bowel prep to see if information could be gleaned to advise future patients.

Methods The study comprised 100 patients having outpatient colonoscopy at St George's Hospital in August to September 2012. In advance of the procedure, patients received two sachets of Citrafleet (to be taken 6 hours apart) and a booklet on colonoscopy \& bowel prep by post. On arrival patients were asked to complete a questionnaire, including information on usual bowel habit, fluid intake, perceived efficacy of the prep and whether the first or second sachet of prep worked better. Patient demographics and medical history were collected by nursing staff at admission clerking. The Endoscopist assessed the quality of bowel prep at colonoscopy using a standard four point score $(0=$ good, $1=$ satisfactory, $2=$ poor, $3=$ very poor $)$.

Results Complete data were collected on 89 patients (age range 21-100; mean age 64): One procedure was abandoned due to failed intubation; in 10 cases the endoscopist did not comment on quality of prep. Endoscopists reported 23 cases where preparation was suboptimal, in contrast to only 5 patients (fifty seven patients felt the laxative worked very well and 38 quite well). There was a tendency towards those who thought the prep worked very well having better prep at colonoscopy, but this did not reach statistical significance $(p=0.24)$. Patients with depression had poorer preparation at colonoscopy $(p<0.01)$.

Fifty seven patients felt their bowels opened most after the first sachet, 43 after the second. Those who thought the second sachet was more effective than the first had poorer bowel prep at colonoscopy $(p=0.03)$. Patients who reported their usual bowel habit as hard, and those with diabetes, had a tendency towards having their bowels open most after the second sachet, but neither relationship was statistically significant ( $p=0.15 \& 0.18$ respectively).

No correlations were found between patient gender, age or drinking habits and perceived quality of prep or efficacy of each sachet.

Conclusion Our results show that patients reporting more effective results after the second sachet of laxative have objectively poorer preparation at colonoscopy. We suggest that this information could be used to advise such patients that an increased fluid intake may be necessary before and after the second dose of laxative to optimise preparation. A larger study may help to identify further correlates with which we can advise our patients.

\section{Disclosure of Interest None Declared.}

\section{REFERENCE}

1. Ness RM, Manam R, Hoen H, Chalasani N. Predictors of inadequate bowel preparation for colonoscopy. Am J Gastroenterol. 2001 Jun; 96(6):1797-802.

\section{PTH-119 NURSES AND ENDOSCOPISTS ARE EQUALLY GOOD AT GAUGING PATIENT DISTRESS DURING ENDOSCOPY}

doi:10.1136/gutjnl-2013-304907.606

${ }^{1}$ A J Irvine, 1,"M Kurien, ${ }^{1} \mathrm{R}$ Harrold, 'L Taylor, 'D S Sanders. 'Department of Gastroenterology, Royal Hallamshire Hospital, Sheffield, UK

Introduction Endoscopic procedures have the potential to be uncomfortable and distressing. Previous studies have suggested nurses are better than endoscopists at gauging patient's pain during endoscopy, possibly reflecting the endoscopists focus on the procedure as opposed to the nurse whose focus remains on the patient. Currently, there is a paucity of work evaluating distress another important marker of endoscopic tolerability. This study evaluates endoscopists' and nurses' ability to gauge patient's distress during gastrointestinal endoscopic procedures.

Methods Consecutive patients attending for clinically indicated gastrointestinal endoscopy were prospectively recruited from a tertiary referral centre between September 2011 and June 2012. Following informed consent being obtained patients were asked to record distress post endoscopy using a validated numeric rating scale (NRS), with scores recorded between 0 (no distress) and 10 (worst distress imaginable). Endoscopists undertaking the procedure and their assisting nurses were then asked to give their estimates of patient's distress using the same NRS, with recordings undertaken separately so as not to influence potential outcomes. Data was analysed using SPSS version 20 , with a correlation coefficient used to determine levels of agreement in distress scores. 